\title{
Detection of Corrosion and Wall Thinning in Carbon Steel Pipe Covered With Insulation Using Pulsed Eddy Current
}

\author{
Duck-Gun Park $^{1 *}$, M. B. Kishore' ${ }^{1}$, J. Y. Kim² , L. J. Jacobs², and D. H. Lee ${ }^{1}$ \\ ${ }^{1}$ Korea Atomic Energy Research Institute, Yuseong, Daejeon 34057, Korea \\ ${ }^{2}$ Georgia Institute of Technology, Atlanta, GA30332-0360, U.S.A
}

(Received 1 December 2015, Received in final form 16 February 2016, Accepted 17 February 2016)

\begin{abstract}
Non Destructive Testing (NDT) methods that are capable of detecting the wall thinning and defects through insulation and cladding sheets are necessary. In this study we developed a Pulsed Eddy Current (PEC) system to detect wall thinning of ferro magnetic steel pipes covered with $95 \mathrm{~mm}$ thick fiber glass thermal insulator and shielded with aluminum plate of thickness $0.4 \mathrm{~mm}$. In order to confirm the thickness change due to wall thinning, two different sensors, a hall sensor and a search coil sensor were used as a detecting element. In both the cases, the experimental data indicates a considerable change in the detected pulse corresponding to the change in sample thickness. The thickness of the tube was made to change such as $2.5 \mathrm{~mm}, 5 \mathrm{~mm}$ and $8 \mathrm{~mm}$ from the inner surface to simulate wall thinning. Fast Fourier Transform (FFT) was calculated using window approach and the results were summarized which shows a clear identification of thickness change in the test specimen by comparing the magnitude spectra.
\end{abstract}

Keywords : wall-thinning, Pulsed Eddy Current, carbon steel, Fast Fourier Transform, hall sensor, search coil

\section{Introduction}

The pipelines of power plants and heat exchangers are covered with a thermal insulator to reduce the heat loss. During the long-term services, corrosion might occur on the outer side of the pipe as Corrosion Under Insulation (CUI), or on the inner side of a pipe as Flow Accelerated Corrosion (FAC) and development into wall thinning of the pipelines is a real threat for the reliability of carbon steel equipment, which may finally results into a catastrophic failure. Therefore, local wall thinning is a point of concern in almost all steel structures. Usually, thermal insulators used for the purpose of covering the pipelines is made up of materials with low thermal conductivity (fiberglass or mineral wool) and externally gladded aluminum or galvanized steel. Hence, NDT methods that are capable of detecting the wall thinning and defects without removing the insulation or through this insulation and cladding are necessary. PEC nondestructive testing is widely used for the characterization of deep flaws in ferromagnetic materials. The conventional ECT which

CThe Korean Magnetics Society. All rights reserved.

*Corresponding author: Tel: +82-42-868-2023

Fax: +82-42-868-4785, e-mail: dgpark@kaeri.re.kr operates with a single frequency sinusoidal excitation has gained wide acceptance in the field of NDT [1]; yet, this technique suffers from a limitation, i.e., penetration depth or skin depth. The penetration depth $\delta$ depends on excitation frequency $\mathrm{f}$ [2]. In contrast to the traditional ECT, the PEC employs a non sinusoidal excitation, such as a pulse or square wave, instead of a single frequency sinusoidal excitation, because the Fourier transform of a pulse contains multiple frequency components $[3,4]$, a rectangular pulse can provide the depth profile of a material under test $[5,6]$. The usage of short current pulse excitation reduces the power consumption, which is the most desired specification for the development of portable instruments. Due to the potential advantages of the PEC, prevalent investigations on this technique have been conducted, such as detection of wall thinning and corrosion in aircraft multilayer structures [7, 8]. The developed PEC system is optimized for the detection of thickness change as well as sub-surface crack detection on the Ferro magnetic samples. A Lab-VIEW based program was used to record the data and to analyze the recorded data. We conducted two different experiments simultaneously to maintain the same experimental conditions and both results were summarized for the thickness variations of the tested sample. Both the results give a concrete change 


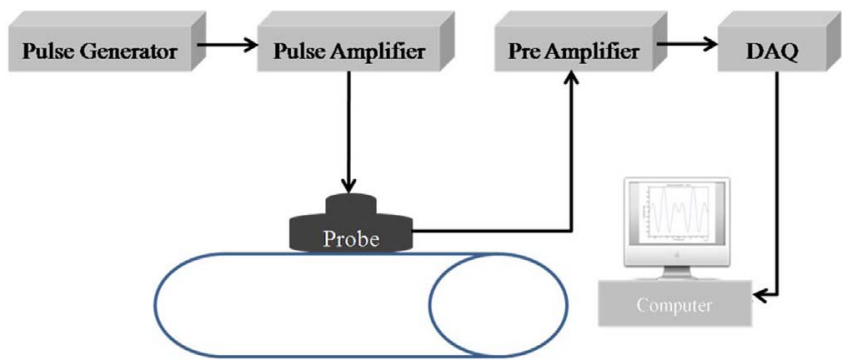

Fig. 1. (Color online) Schematic diagram of the PEC system.

in the thickness of the sample

\section{System Designing}

The PEC system consists of a pulse generator, a pulse amplifier, a probe with a driving coil and a magnetic field detecting sensor, a sensitive PEC preamplifier with variable gain to amplify the output voltage from the magnetic field detection sensor, a data acquisition system to convert analog data to digital data and log the data, and a computer with instrument controlling and processing signals. Fig. 1 shows the schematic diagram of the designed PEC system. A pulse amplifier inputted from a pulse generator will drives the excitation coil of the PEC probe and the response signals obtained from Hall sensor and search coil will be given to a sensor electronic or preamplifier. The proper conditioned signal from preamplifier is fed to DAQ system and recorded in computer connected through a software application. A typical PEC probe is consisted of an exciting coil and a magnetic field detection sensor. The excitation coil is made up of winding a copper wire on a core or air core bobbin, and detection coil is wound around the excitation coil. A commercially available hall sensor with proper connections was placed at the center of the exciting coil to detect the response from eddy currents. A real-time Lab-VIEW program was developed for data acquisition. The probe was scanned on the test sample to check the thickness of the sample and the analyzed results were displayed on the computer monitor.

\section{Experimental Setup}

During these experiments a ferro magnetic steel tube of diameter $210 \mathrm{~mm}$ and a length of $620 \mathrm{~mm}$, which is covered with an insulator of $95 \mathrm{~mm}$ thickness was used as a wall thinned pipe with various thickness regions 2.5 $\mathrm{mm}, 5 \mathrm{~mm}$ and $8 \mathrm{~mm}$ respectively. Fig. 2(c) depicts the inner cross section of the tube covered with insulation and shielded with aluminum plate. A $95 \mathrm{~mm}$ thick insulator with galvanized cladding of $0.4 \mathrm{~mm}$ thickness covers the
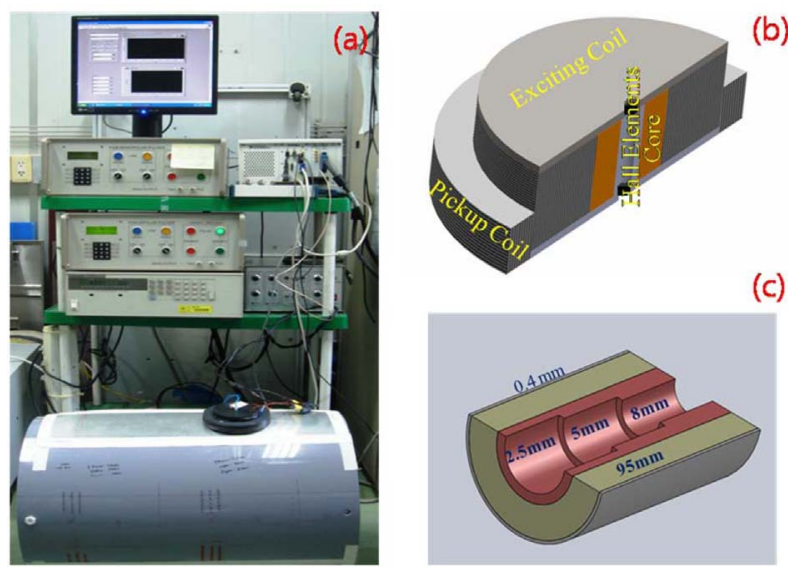

(b)

(c)

Fig. 2. (Color online) (a) Experimental setup of PEC system, (b) Cross sectional view of probe, (c) Inner cross sectional view of the test specimen.

pipeline to simulate real time environment. A bipolar rectangular current pulse generated from pulse amplifier was used to excite the PEC probe and the detected sensor output is fed into the PEC amplifier for signal conditioning. The design of PEC probe is shown in Fig. 2(b), a copper wire wound on a ferrite core is used to exert magnetic field into the test specimen consequently generating eddy currents in the test sample, the eddy currents response generated from test specimen will be detected by two sensors simultaneously i.e. a Hall sensor placed at the center of the ferrite core and a pickup coil sensor wounded around the exciting coil. The preamplifier will signal conditioning and preprocess the detected pulse so that it can be logged by the DAQ system connected to a computer through DAQ interface as shown in the Fig. 2(a). While placing the probe great care was taken to avoid the ambiguity in the results of thickness change. The probe was scanned along the different thickness segments of pipe. First the Probe was placed on $2.5 \mathrm{~mm}$ thick region of the pipe, the excited pulse was repeated for five times and the average detected response from Hall sensor and coil sensor for five pulses was recorded as the resultant detected pulse corresponding to $2.5 \mathrm{~mm}$ thickness which leads to reduce the percentage of error as less as possible and improves the signal to noise ratio, a desired characteristic in measurement process. After finishing the measurement, the probe was moved to $5 \mathrm{~mm}$ thick region of the sample and repeated the same process to record the detected signal corresponding to $5 \mathrm{~mm}$ thickness. Finally, the probe was moved to $8 \mathrm{~mm}$ thickness region and its corresponding detected pulse is recorded in same fashion. All the data recording process has been controlled by a program developed in Lab VIEW environment also the data analysis and processing can be 
done by using developed program. Once after completing the data recording process all the data will be analyzed and displayed on the computer. The system applied in the mock-up can distinguish a wall thickness of $2.5,5$, and 8 $\mathrm{mm}$ under $95 \mathrm{~mm}$ insulation covered with $0.4 \mathrm{~mm}$ of aluminum cladding.

\section{Results and Analysis}

When a high-level current is applied to the exciting coil, the coil makes a magnetic field (primary magnetic field) into the sample; when there is a sudden transition in the exciting current, eddy currents are induced into the sample. The time-varying magnetic fields (secondary magnetic field) generated by the decaying eddy currents are captured by the Hall-sensor as a voltage signal. The strength and duration of induced pulse signal resembles the average wall thickness that can be measured. The duration of the eddy currents will have an effect on the transition period of the detected pulse. Form the Fig. 3(a) it is clearly observed that the transition time gradually increases as the thickness of the sample increases during the rising of the detected pulse. In other words the rising edge of the detected pulse is delayed more as the thickness of the sample increases, during its falling edge the delay is decreasing as the thickness increases also the magnitude of the detected pulse gradually decreases with increasing sample thickness. When the insulation over the test specimen is covered with $0.4 \mathrm{~mm}$ aluminum plate, same phenomenon is observed i.e. the transition period gradually increases as the thickness of the sample increases during the rising of the detected pulse, also the magnitude of the detected pulse gradually decreases with increasing sample thickness in the detected pulse. The detected pulse is further reduced compared to the previous case i.e. without aluminum plate on the insulation as shown in Fig. 3(b). The slope of rising pulse in the Al shielded one increased compared to that of unshielded one, which is attributed to the damping of the eddy current by Al shield.

The signal obtained from Hall sensor was performed with FFT analysis using rectangular type window approach and the results were summarized as shown in the Fig. 4. It is a clear evidence that, at lower frequencies, the magnitude spectra of the detected pulse indicates a gradual decrease in magnitude as the sample thickness is increasing from $2.5 \mathrm{~mm}$ to $8 \mathrm{~mm}$. The frequencies also shift to a lower frequency region with increasing sample thickness, which attributed to the eddy current damping associated with skin depth. The eddy current density is more and present on the surface of the sample if the sample thickness is less and gradually decreases with increasing sample

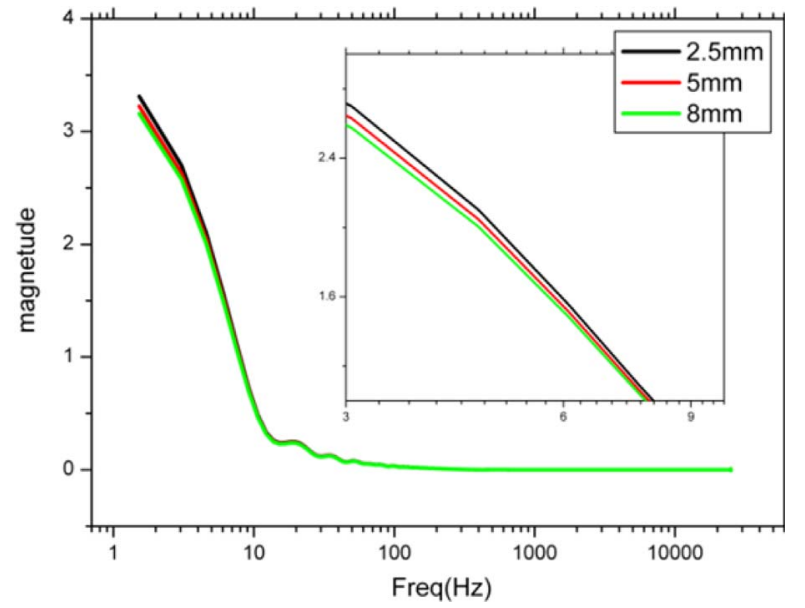

Fig. 4. (Color online) Magnitude spectra of the response signal obtained from Hall Sensor.
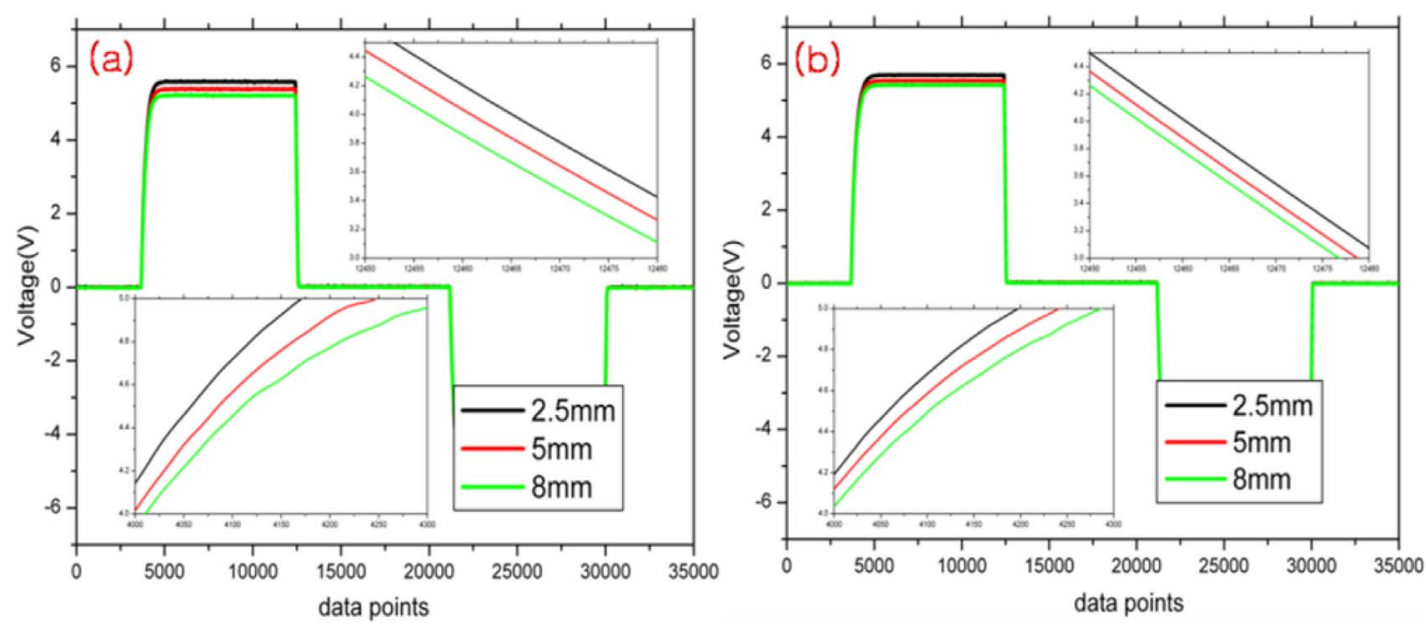

Fig. 3. (Color online) (a) Hall Sensor output with $95 \mathrm{~mm}$ insulation and no aluminum shielding, (b) Hall sensor output when the sample insulated with $95 \mathrm{~mm}$ insulation and shielded with $0.4 \mathrm{~mm}$ aluminum plate. 

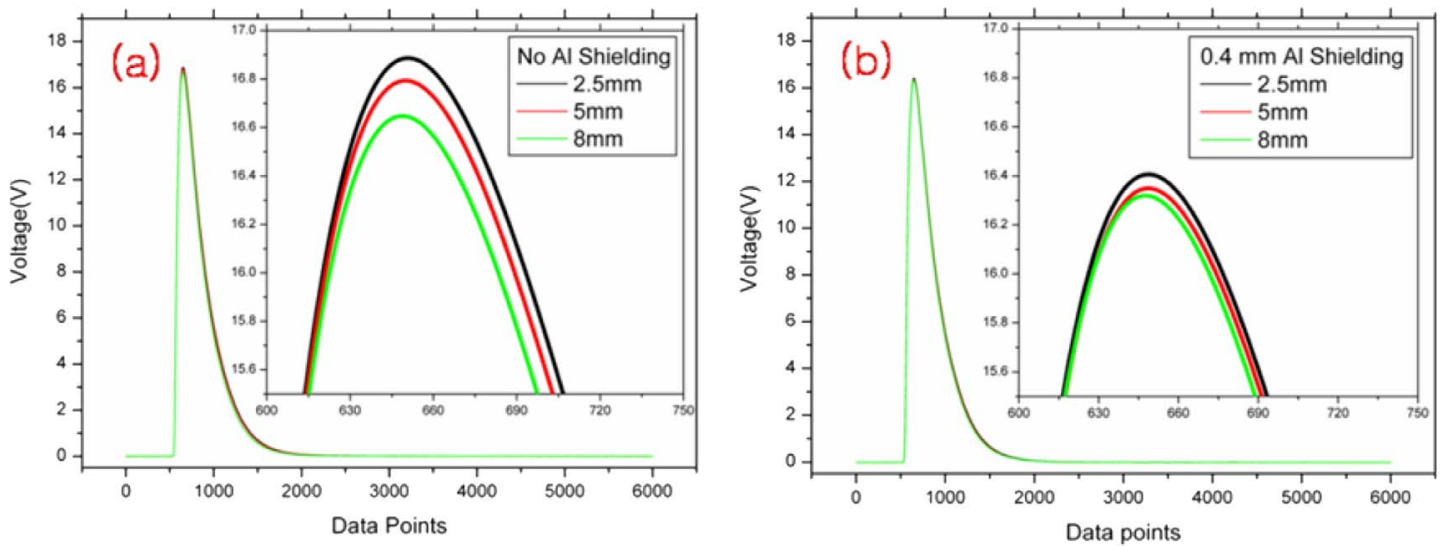

Fig. 5. (Color online) (a) Coil Sensor output with $95 \mathrm{~mm}$ Insulation and no aluminum shielding, (b) Coil Sensor output when the sample insulated with $95 \mathrm{~mm}$ Insulation and shielded with $0.4 \mathrm{~mm}$ aluminum plate.

thickness resulting into a small decrease in magnitude. The magnitude decreases gradually as the frequency increases but the decrement rate is more for $8 \mathrm{~mm}$ thick region rather than $2.5 \mathrm{~mm}$ causing a shift to lower frequency region. The eddy currents decays at a faster rate as the thickness increases, which means that the energy of the detected pulse for higher thickness region is small compared to that of lower thickness regions.

To confirm the Hall sensor results, search coil sensor results are also analyzed which concludes the same as that of hall sensor detection method. Since both the experiments are conducted in a single instant all the experimental conditions are exactly same as the prior experiment which is clearly shown in Fig. 5(a). From this figure it is clear that the peak of the sensing signal gradually increases as the thickness of the tube decreases, it is observed that the decay of the eddy currents increases as the thickness decreases which is a clear identification of the change in the sample thickness. When the insulation on the sample is shielded with Aluminum plate the magnitude of the detected pulse considerably decreased since the penetration of the excited magnetic fields are limited by the aluminum shielding as shown in Fig. 5(b).

\section{Conclusion}

The PEC technique which can detects wall thinning of pipelines covered with thick insulation and shielded with thick metal sheets was developed using a Hall sensor and search coil sensor. The probe performance was tested using the wall thinning pipe covered with $95 \mathrm{~mm}$ insulation covered with $0.4 \mathrm{~mm}$ of aluminum cladding. The results obtained from Hall-sensor show better resolution than that of search coil sensor. The detected pulse is further reduced compared without aluminum plate on the insulation. The slope of rising pulse in the $\mathrm{Al}$ shielded one increased compared to that of unshielded one, which is attributed to the damping of the eddy current by $\mathrm{Al}$ shield. The eddy currents decays at a faster rate as the thickness increases, which means that the energy of the detected pulse for higher thickness region is small compared to that of lower thickness regions. We can discriminate the thickness of the test specimen from this frequency spectrum of induced pulse signal.

\section{References}

[1] N. Nair, V. Melapudi, J. Hector, X. Liu, Y. Deng, Z. Zang, L. Udpa, J. M. Thomas, and S. Udpa, IEEE Trans. Magn. 42, 3312 (2006).

[2] R. Griberg, L. Udpa, A. Savin, R. Steigmann, V. Palihovic, and S. S. Udpa, NDT\&E Int. 39, 264 (2006).

[3] Sophian, G. Y. Tian, D. Taylor, and J. Rudlin, NDT \& E int. 36, 37 (2003).

[4] Zhang Gang and Zhao Liang, Transducer and Microsystems Technologies 25, 35 (2006).

[5] C. J. Renken. Materials Evaluation 59, 356 (2001).

[6] J. Blitz, Electrical and Magnetic Method of Non-Destructive Testing, Chapman \& Hall, London (1997).

[7] M. A. Robers and R. S. Scottini, Proc. of 8th ECNDT conf, ndt.net, Barcelona, 7, (2002).

[8] R. A. Smith and G. R. Hugo, Insight 43, 14 (2001). 